\title{
Mensurações do bulbo ocular e cálculo do poder dióptrico de lentes intraoculares em coelhos
}

\author{
[Measurements of ocular bulb and calculation of the dioptric power of intraocular \\ lenses in rabbits]
}

\author{
M.A.R. Valinhos $^{1}$, J.J.T. Ranzani ${ }^{1}$, A.C.L. Rodrigues ${ }^{2}$, C.V.S. Brandão ${ }^{1}$ \\ ${ }^{1}$ Faculdade de Medicina Veterinária e Zootecnia - UNESP - Botucatu, SP \\ ${ }^{2}$ Faculdade de Medicina - UNESP - Botucatu, SP
}

\begin{abstract}
RESUMO
Determinaram-se as dimensões oculares de coelhos, empregando ultrassonografia modo-A e ceratometria, a fim de utilizá-las como subsídios para calcular o poder dióptrico de lentes intraoculares a serem implantadas experimentalmente nessa espécie e avaliar se existe diferença entre as mensurações oculares de machos e fêmeas e entre olho esquerdo e olho direito. Foram utilizados 58 animais do grupo genético Botucatu, distribuídos entre 30 fêmeas e 28 machos, com idade média de 85,8 88,29 dias e peso médio de $2,3 \pm 0,46 \mathrm{~kg}$. O cálculo da lente intraocular foi realizado por meio do software Holladay IOL Consultant ${ }^{\circledR}$. As fórmulas empregadas para o cálculo foram: Holladay II, Holladay I, Hoffer Q e SRK-T, que calcularam lentes de aproximadamente 48,5 D; 50,27 D; 59,67 D e 49,24 D, respectivamente. A fórmula SRK-T mostrou-se semelhante às fórmulas Holladay II e Holladay I, enquanto entre as demais ocorreram diferenças. As características biométricas oculares avaliadas, assim como o poder dióptrico calculado por uma mesma fórmula, não apresentaram diferenças significativas com relação ao sexo e à lateralidade.
\end{abstract}

Palavras-chave: coelho, biometria, ceratometria, lente intraocular

\begin{abstract}
The rabbit ocular dimensions were determined using A-mode ultrasound and keratometry, in order to use them as data for calculating the dioptric power of intraocular lenses to be implanted experimentally in this species, as well as assess whether there are differences in ocular measurements between males and females and between the left eye and right eye in rabbits. Fifty-eight animals ( 30 females and 20 males) of the Botucatu genetic group, with a mean age of $85.8 \pm 8.29$ days and average weight of $2.3 \pm 0.46 \mathrm{~kg}$ were used. The calculation of the intraocular lens was performed using Holladay IOL Consultant ${ }^{\circledR}$. The formulas used for the calculation were: Holladay II, Holladay I, Hoffer $Q$ and SRK-T which estimated lenses around $48.5 \mathrm{D}, 50.27 \mathrm{D}, 59.67 \mathrm{D}$ and $49.24 \mathrm{D}$, respectively. The SRK-T was statistically similar to the Holladay II and Holladay I, while the others were different from each other. The ocular biometric parameters evaluated, as well as the lens power calculated by the same formula showed no significant differences in relation to gender and laterality.
\end{abstract}

Keywords: rabbit, biometry, keratometry, intraocular lens

\section{INTRODUÇÃO}

O tratamento da catarata é eminentemente cirúrgico e implica na remoção do cristalino (facectomia). Porém, a remoção dessa poderosa lente convergente faz com que o olho afácico sem cristalino - passe a contar com o poder refrativo apenas da córnea, tornando-se altamente hipermétrope - com ponto focal atrás da retina (Gaiddon et al., 1991). A correção da afacia pode ser feita mediante o uso de lentes intraoculares (LIOs), atualmente empregadas como rotina nas cirurgias de catarata em humanos.

Recebido em 22 de março de 2011

Aceito em 13 de julho de 2011

E-mail: mayanavalinhos@yahoo.com.br 
$\mathrm{Na}$ oftalmologia humana, diversas fórmulas têm sido preconizadas para o cálculo do poder dióptrico adequado dos implantes, visando obter um olho emétrope. Durante muitos anos, o objetivo da cirurgia de catarata, na oftalmologia veterinária, foi a recuperação da capacidade visual, sendo considerada satisfatória pelos cirurgiões a visão afácica hiperópica dos seus pacientes, que permite evitar colisões e reconhecer pessoas e objetos. No entanto, é crescente a preocupação em promover uma visão o mais emétrope possível para os animais submetidos à facectomia, acreditando-se que isto possa oferecer melhor qualidade de vida para eles. Porém, o cálculo do poder dióptrico da lente a ser implantada em animais ainda é um assunto pouco explorado. Há alguns trabalhos utilizando as fórmulas humanas disponíveis, porém ainda sem conclusões bem estabelecidas sobre a sua precisão quando aplicadas em olhos de animais.

A utilização de coelhos como modelo experimental, em detrimento dos cães, tem sido cada vez mais frequente, por questões éticas e de facilidade de manejo. Esse fato justifica, portanto, a necessidade de um conhecimento mais detalhado sobre sua morfologia ocular a fim de adequar técnicas, como por exemplo, o cálculo do poder dióptrico de lentes intraoculares para esses animais, possibilitando a continuidade e a evolução dos estudos sobre este assunto de relevância em oftalmologia veterinária.

O presente trabalho teve como objetivos calcular o poder dióptrico de lentes intraoculares em coelhos, por meio de fórmulas desenvolvidas para olhos humanos, utilizando subsídios métricos obtidos por meio de biometria e ceratometria, assim como avaliar se existe diferença entre as mensurações oculares de machos e fêmeas, bem como entre olho esquerdo e olho direito em coelhos.

\section{MATERIAL E MÉTODOS}

Os procedimentos experimentais desenvolvidos foram submetidos à Câmara de Ética em Experimentação Animal da Faculdade de Medicina Veterinária e Zootecnia, UNESP, Botucatu (Protocolo no 15/2009 - CEEA).

Foram utilizados 58 animais (116 olhos) da espécie leporina (Oryctolagus cuniculus) e do grupo genético Botucatu, sendo 30 fêmeas e 28 machos, sadios ao exame clínico e oftálmico. A idade média das fêmeas foi de 89,40 $\pm 9,31$ dias, com peso médio de $2,07 \pm 0,31 \mathrm{~kg}$; e dos machos $82,50 \pm 5,56$ dias, com peso médio de $2,46 \pm 0,51 \mathrm{~kg}$. Utilizou-se apenas contenção mecânica manual dos coelhos, sendo suficiente para realização dos exames.

O diâmetro corneano, ou seja, medida branco-abranco horizontal, foi mensurado com auxílio de compasso cirúrgico (Compasso Castroviejo reto) e anestesia tópica prévia, com colírio de cloridrato de proximetacaína

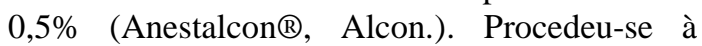
mensuração da curvatura corneana (ceratometria) com a utilização de um autoceratômetro (KM500, Nidek Co.). Foram realizadas três aferições consecutivas em cada olho, obtendo-se um valor médio de K1 (valor do menor meridiano da córnea), K2 (valor do maior meridiano da córnea) e K (média entre K1 e K2) para cada indivíduo.

A técnica ultrassonográfica modo-A empregada foi a de aplanação, com aplicação prévia de colírio de cloridrato de proximetacaína $0,5 \%$, por meio de um biômetro ultrassônico (NIDEK Echoscan Model US-800, Nidek Co.) em modo automático, cujo transdutor de $10 \mathrm{MHz}$ foi posicionado em contato direto com a córnea e alinhado ao eixo óptico. O aparelho foi calibrado com as velocidades de $1.731 \mathrm{~m} / \mathrm{s}$ para o cristalino e $1.534 \mathrm{~m} / \mathrm{s}$ para humor aquoso e vítreo (Görig et al., 2006).

Foi considerada a média de seis mensurações do comprimento axial do bulbo ocular, profundidade de câmara anterior, espessura do cristalino e comprimento axial da câmara vítrea. $\mathrm{O}$ cálculo da lente intraocular foi realizado para ambos os olhos de cada indivíduo, por meio do software Holladay IOL Consultant ${ }^{\circledR}$ (Holladay IOL Consultant Software - Professional Edition.). Foram fornecidas ao software variáveis individuais de acordo com o olho em questão, como comprimento axial, profundidade de câmara anterior e espessura do cristalino, ceratometria (meridianos K1 e K2), medida branco-a-branco horizontal (diâmetro corneano), sendo considerada, para todos os indivíduos, a técnica de implantação da lente no saco capsular, a refração alvo plana, a refração pré-operatória plana e a lente (Ioflex Mediphacos®) com constante A de 118 fornecida pelo fabricante. 
As fórmulas empregadas para o cálculo foram: Holladay II, Holladay I, Hoffer Q e SRK-T. Os dados foram submetidos à técnica de análise de variância para o modelo de medidas repetidas em grupos independentes, complementada com os respectivos testes de comparações múltiplas (Zar, 1999). O nível de significância considerado foi de $5 \%$

\section{RESULTADOS E DISCUSSÃO}

Para que a lente intraocular atinja o objetivo de aproximar o paciente facectomizado da emetropia, seu poder dióptrico deve ser calculado corretamente. A acurácia do cálculo depende basicamente de três fatores: precisão dos dados biométricos, rigidez do controle de qualidade do fabricante da LIO e previsibilidade da fórmula empregada (Hoffer, 1993; Narváez et al., 2006).

A implantação de LIOs na oftalmologia veterinária para correção da afacia é um assunto de crescente interesse no Brasil, porém ainda restrito a condições experimentais. Na literatura estrangeira, no entanto, aparece como um procedimento já bem estabelecido em cães (Gaiddon et al., 1997; Gilger et al., 1998; Kecová e Necas, 2004; McMullen et al., 2010), todavia sem conclusões bem definidas sobre como calcular o poder dióptrico corretamente e sem dados objetivos sobre a real vantagem da utilização das lentes intraoculares. Para isso, são necessários mais estudos com implantação de LIOs para obtenção de maior número de dados pós-operatórios de animais, possibilitando a análise da previsibilidade das diversas fórmulas humanas disponíveis, o que pode ser facilitado pelo uso de modelos experimentais com o coelho.

A utilização de coelhos de ambos os sexos teve como objetivo a verificação de possível influência desta variável sobre as mensurações oculares. Em 1982, Schiffer et al. relataram uma diferença estatística do comprimento axial entre os olhos de machos e fêmeas da espécie canina. No estudo de Bozkir et al. (1997), os coelhos machos apresentaram poder de refração da córnea e comprimento axial do bulbo ocular maiores que as fêmeas. No entanto, Sampaio et al. (2002) constataram que não há diferença significativa do comprimento axial dos olhos e da curvatura corneana entre machos e fêmeas da espécie canina, sendo esta consideração condizente com os achados deste estudo.

Foram realizadas mensurações separadas para olho direito e olho esquerdo, pois esta variável poderia influenciar os resultados finais. Schiffer et al. (1982) não encontraram diferença entre o comprimento axial dos olhos direitos comparados aos esquerdos, em estudo biométrico de olhos de cães utilizando ultrassonografia modo-A. Sampaio et al. (2002) também concluíram não haver diferença significativa do comprimento axial e da ceratometria entre os olhos direito e esquerdo de cães, assim como Uthoff (1984) em coelhos, o que foi comprovado mais uma vez por este trabalho.

O comprimento axial dos bulbos oculares dos machos foi de $15,54 \pm 0,66 \mathrm{~mm}$ e das fêmeas foi de 15,65 $\pm 1,41 \mathrm{~mm}$; em olho esquerdo observou-se $15,56 \pm 1,01 \mathrm{~mm}$, e em olho direito $15,63 \pm 1,19 \mathrm{~mm}$. Estes dados assemelham-se aos $16,3 \mathrm{~mm}$ e $16,2 \mathrm{~mm}$ para olhos direito e esquerdo, respectivamente, relatados por Uthoff (1984) e por Bozkir et al. (1997), que citaram $15,33 \pm 0,50 \mathrm{~mm}$ em machos e $14,96 \pm 0,46 \mathrm{~mm}$ em fêmeas. Os valores deste estudo assemelham-se também aos de Werner et al. (2006), que observaram comprimento axial médio de $16.6 \pm 0,48 \mathrm{~mm}$

A espessura média do cristalino foi de $6,02 \pm 0,58 \mathrm{~mm}$. Werner et al. (2006) relataram valor semelhante de $6,36 \pm 0,13 \mathrm{~mm}$, porém as mensurações de Uthoff (1984) foram inferiores às deste estudo, uma vez que o autor observou $4,8 \mathrm{~mm}$ em olho direito e $4,9 \mathrm{~mm}$ em olho esquerdo. Com relação à profundidade de câmara anterior, o valor médio encontrado foi de $2,56 \pm 0,14 \mathrm{~mm}$, resultado semelhante aos $2,7 \mathrm{~mm}$ observados em ambos os olhos por Uthoff (1984) e aos $2,16 \pm 0,11 \mathrm{~mm}$ relatados por Werner et al. (2006). Os estudos citados anteriormente não investigaram o comprimento da câmara vítrea, impossibilitando a comparação dos achados deste estudo com esses outros.

Os valores médios obtidos para ceratometria foram 49,66 $\pm 2,06 \mathrm{D}$ ou $6,81 \pm 0,29 \mathrm{~mm}$ em olho esquerdo e 49,76 $\mathrm{D} \pm 2,01$ ou $6,79 \pm 0,28 \mathrm{~mm}$ em olho direito, sendo superiores em aproximadamente 5D aos valores relatados por Uthoff (1984), porém próximos aos $7,26 \mathrm{~mm}$ encontrados por Bozkir et al. (1997). 
Constatou-se que o diâmetro corneano, em torno de $12,6 \mathrm{~mm}$, foi ligeiramente inferior aos 13,41 mm encontrados por Bozkir et al. (1997) e aos 14,6mm relatados por Uthoff (1984).

Os poderes dióptricos médios da LIO calculados pelas fórmulas de terceira geração Holladay I, SRK-T e Hoffer-Q foram, respectivamente, 50,27 D, 49,24 D e 59,67 D. Não houve diferença estatística entre os valores calculados pela Holladay I e SRK-T, o que está de acordo com a afirmação de Prado-Serrano e NavaHernández (2009) sobre o comportamento preditivo semelhante das fórmulas de terceira geração, com exceção dos olhos curtos, nos quais a fórmula Hoffer-Q sempre calcula LIOs mais potentes (Prado-Serrano e Nava-Hernández, 2009) .

A fórmula de quarta geração Holladay II demonstrou um valor dióptrico médio de 48,5 D. No entanto, não houve diferença desta em relação à formula SRK-T.

$\mathrm{Na}$ oftalmologia humana, após diversos estudos comparativos, concluiu-se que a previsibilidade das fórmulas está intimamente relacionada ao comprimento axial do olho no qual é empregada. $\mathrm{Na}$ oftalmologia veterinária, no entanto, pouco se tem estudado sobre a previsibilidade das fórmulas quando aplicadas em olhos animais, não havendo, portanto, um consenso de como deve ser feita a eleição da fórmula.

A maioria dos estudos relacionados ao cálculo de lentes intraoculares para correção da afacia em animais empregou fórmulas humanas de primeira geração (Gaiddon et al., 1991; Gilger et al., 1998b; Sampaio et al., 2002; McMullen e Gilger, 2006) e não implantou a lente calculada para avaliar o erro refrativo pós-operatório e verificar o grau de previsibilidade da fórmula utilizada. Outros estudos foram realizados com a fórmula de terceira geração SRK-T, a partir dos quais concluiu-se que ela não ofereceu bons resultados em cães (Mobricci, 2006; Peixoto et al., 2008). O resultado insatisfatório obtido por estas fórmulas, provavelmente, deve-se ao fato de que a proporção seguimento anterior, seguimento posterior e espessura do cristalino nos animais não é a mesma do olho humano (Peixoto et al., 2008) e por isso estas fórmulas estimam uma ELP (posição efetiva da lente) errada. Em humanos isto ocorre em olhos com comprimento axial menor que $18,0 \mathrm{~mm}$, chamados extremamente curtos (Holladay et al., 1996).

Sabe-se que olhos humanos maiores que 18,0 $\mathrm{mm}$ de comprimento axial mantém constante proporção segmento anterior/segmento posterior, ou seja, olhos curtos têm seguimentos anterior e posterior curtos, e olhos longos têm segmentos anterior e posterior longos. No entanto, olhos extremamente curtos com comprimento axial menor que $18,0 \mathrm{~mm}$ não mantêm esta relação, podendo ter segmentos anterior e posterior curtos, segmento anterior normal e posterior curto ou segmento anterior curto e posterior normal, perdendo, assim, as proporções e tornando inadequado o cálculo feito pelas fórmulas de terceira geração (Holladay et al., 1996).

Para estes olhos, a fórmula indicada é a Holladay II, pois, enquanto as fórmulas de terceira geração utilizam apenas a ceratometria e o comprimento axial para estimar a ELP, a Holladay II individualiza o olho usando mais parâmetros, como diâmetro corneano, tamanho da câmara anterior e espessura do cristalino, para fazer esta estimativa, passando a não depender da relação habitual entre segmentos anterior e posterior para ser precisa. Seguindo este raciocínio, espera-se que esta fórmula seja mais adequada para utilização em olhos de animais, que não guardam as mesmas proporções entre segmento anterior e segmento posterior do olho humano.

Além de o olho do coelho corresponder a um olho humano classificado como extremamente curto $(<18 \mathrm{~mm})$, ele não guarda as mesmas proporções do olho humano (Werner et al., 2006). No entanto, o presente estudo não mostrou diferença estatística entre os poderes dióptricos da LIO calculados pela Holladay II e pela fórmula de terceira geração SRK-T. Possíveis justificativas para a semelhança entre as duas fórmulas podem ser o tamanho da amostra ou o fato de a Holladay II não apresentar previsibilidade superior à SRK-T em olhos de coelhos, como se havia imaginado.

A verificação da previsibilidade das fórmulas e da superioridade da Holladay II sobre as fórmulas de terceira geração em olhos de animais depende da implantação das lentes calculadas e da avaliação do erro refracional pós-operatório, ou do conhecimento da ELP pós-implante, que 
permitiria o cálculo exato do poder dióptrico necessário para atingir a emetropia.

\section{CONCLUSÕES}

As características biométricas oculares avaliadas e o poder dióptrico da LIO calculado por uma mesma fórmula não apresentaram diferenças significativas com relação ao sexo e à lateralidade. O poder dióptrico das LIOs a serem implantadas em coelhos para atingir a emetropia varia de acordo com a fórmula utilizada. São necessários mais estudos, com a implantação das lentes calculadas, para avaliação da previsibilidade destas fórmulas em olhos de coelhos.

\section{AGRADECIMENTO}

À Fundação de Amparo à Pesquisa do Estado de São Paulo (FAPESP), pelo apoio financeiro $\left(\mathrm{n}^{\circ}\right.$ 2009/03301-6).

\section{REFERÊNCIAS}

BOZKIR, G.; BOZKIR, M.; DOGAN, H. et al. Measurements of axial length and radius of corneal curvature in the rabbit eye. Acta Med. Okayama, v.51, p.9-11, 1997.

GAIDDON, J.; ROSOLEN, S.G.; STERU, L. et al. Use of biometry and keratometry for determining optimal power for intraocular lens implants in dogs. Am. J. Vet. Res., v.52, p.781-783, 1991.

GAIDDON, J.; LALLEMENT, P.E.; BOUHANA, N. Study of refraction by retinoscopy of normal, aphakic and pseudophakic canine eyes: advantages of using a 41 diopter intraocular lens. Prat. Med. Chir. Anim. Comp., v.32, p.531-535, 1997.

GILGER, B.C.; DAVIDSON, M.G.; HOWARD, B.S. Keratometry, ultrasonic biometry, and prediction of intraocular lens power in the feline eye. Am. J. Vet. Res., v.59, p.131-134, 1998.

GÖRIG, C.; VARGHESE, T.; STILES, T. et al. Evaluation of acoustic wave propagation velocities in the ocular lens and vitreous tissues of pigs, dogs, and rabbits. Am. J. Vet. Res., v.67, p.288-294, 2006.

HOFFER, K.J. The Hoffer Q formula: A comparision of theoretic and regression formulas. J. Cataract Refract. Surg., v.19, p.700-712, 1993.

HOLLADAY, J.T.; GILLS, J.P.; LEIDLEIN, J. et al. Achieving emmetropia in extremely short eyes with two piggyback posterior chamber intraocular lenses. Ophthalmology, v.103, p.1118-1123, 1996.
KECOVÁ, H.; NECAS, A. Review Article Phacoemulsification and intraocular lens implantation: recent trends in cataract surgery. Acta Vet. Brno., v.73, p.85-92, 2004.

McMULLEN, R.J.; GILGER, B.C. Keratometry, biometry and prediction of intraocular lens power in the equine eye. Vet. Ophthalmol., v.9, p.357-360, 2006.

McMULLEN, R.J.JR.; DAVIDSON, M.G.; CAMPBELL, N.B. et al. Evaluation of 30- and 25diopters intraocular lens implants in equine eyes after surgical extraction of the lens. Am. J. Vet. Res., v.71, p.809-816, 2010.

MOBRICCI, L.A.L. Avaliação do erro refracional por retinoscopia com luz em faixa em cães fácicos, afácicos e pseudofácicos. 2006. 138f. Tese (Doutorado) - Faculdade de Medicina Veterinária e Zootecnia, Universidade Estadual Paulista, Botucatu, SP.

NARVÁEZ, J.; ZIMMERMAN, G.; STULTING, R.D. et al. Accuracy of intraocular lens power prediction using the Hoffer Q, Holladay 1, Holladay 2, and SRK/T formulas. J. Cataract Refr. Surg., v.32, p.2050-2053, 2006.

PEIXOTO, T.P.; RANZANI, J.J.T.; BRANDÃO, C.V.S. et al. Análise da fórmula SRK/T no cálculo de lente intraocular em cães portadores de catarata. Arq. Bras. Med. Vet. Zootec., v.60, p.1418-1425, 2008.

PRADO-SERRANO, A.; NAVA-HERNÁNDEZ, N.G. Cálculo del poder dióptrico de lentes intraoculares? Cómo evitar la sorpresa refractiva? Rev. Mex. Oftalmol., v.83, p.272280, 2009. Disponível em: <http://www.medigraphic.com/pdfs/revmexoft/rmo2009/rmo095e.pdf>. Acessado em 27 out. 2010.

SAMPAIO, G.R.; RANZANI, J.J.T.; SCHELLINI, S.A. Sexo, peso e conformação anatômica do olho sobre cálculo de poder dióptrico de lentes intraoculares no cão. Cienc. Rural, v.32, p.263-268, 2002.

SCHIFFER, S.P.; RANTANEN, N.W.; LEARY, G.A. et al. Biometric study of the canine eye, using A-mode ultrasonography. Am. J. Vet. Res., v.43, p.826-830, 1982.

UTHOFF, D. Biometrische untersuchungen des kaninchenauges. Klin. Monatsbl. Augenh., v.185, p.189-192, 1984.

WERNER, L.; CHEW, J.; MAMALIS, N. Experimental evaluation of ophthalmic devices and solutions using rabbit models. Vet. Ophthalmol., v.9, p.281-291, 2006.

ZAR, J.H. Biostatistical analysis. 4.ed. New Jersey: Prentice Hall, 1999. 